\title{
Coupling constant limits of Schrödinger operators with critical potentials
}

Xiaoyao $\mathrm{Jia}^{1 *}$ and Yan Zhao ${ }^{2}$

\author{
"Correspondence: \\ jiaxiaoyao@gmail.com \\ ${ }^{1}$ Mathematics and Statistics School \\ Henan University of Science and \\ Technology, No. 263, Luo-Long \\ District, Kai-Yuan Road, Luoyang \\ City, Henan Province 471023, China \\ Full list of author information is \\ available at the end of the article
}

\begin{abstract}
A family of Schrödinger operators, $P(\lambda)=P_{0}+\lambda V$, is studied in this paper. Here $P_{0}=-\Delta+f(x)$ with $f(x) \sim \frac{1}{|x|^{2}}$ when $|x|$ is large enough and $V(x)=O\left(|x|^{-2-\epsilon}\right)$ for some $\epsilon>0$. We show that each discrete eigenvalue of $P(\lambda)$ tends to 0 when $\lambda$ tends to some $\lambda_{0}$. We get asymptotic behavior of the smallest discrete eigenvalue when $\lambda$ tends to $\lambda_{0}$.
\end{abstract}

Keywords: Schrödinger operator; critical potential; asymptotic expansion

\section{Introduction}

In this paper, we consider a family of Schrödinger operators $P(\lambda)$ which are the perturbation of $P_{0}$ in the form

$$
P(\lambda)=P_{0}+\lambda V \quad \text { for } \lambda \geq 0
$$

on $L^{2}\left(\mathbb{R}^{d}\right), d \geq 2$. Here $P_{0}=-\Delta+\frac{q(\theta)}{r^{2}}$. $(r, \theta)$ are the polar coordinates on $\mathbb{R}^{d}$, and $q(\theta)$ is a real continuous function. $V \leq 0$ is a non-zero continuous function satisfying

$$
|V(x)| \leq C\langle x\rangle^{-\rho_{0}} \quad \text { for some } \rho_{0}>2 \text {. }
$$

Here $\langle x\rangle=\left(1+|x|^{2}\right)^{1 / 2}$. Let $\Delta_{s}$ denote the Laplace operator on the sphere $\mathbb{S}^{d-1}$. Assume that

$$
-\Delta_{s}+q(\theta)>-\frac{1}{4}(d-2)^{2} \quad \text { on } L^{2}\left(\mathbb{S}^{d-1}\right) .
$$

If (2) holds, then $P_{0} \geq 0$ in $L^{2}\left(\mathbb{R}^{d}\right)$ (see [1]).

Under the assumption on $V$, we know that $P(\lambda)$ has discrete eigenvalues when $\lambda$ is large enough, and each discrete eigenvalue tends to zero when $\lambda$ tends to some $\lambda_{0}$ (see Section 2). We study the asymptotic behaviors of the discrete eigenvalues of $P(\lambda)$ in this paper. The asymptotic behaviors for Schrödinger operators with fast decaying potentials were studied by Klaus and Simon [2]. In [2], they studied the convergence rate of discrete eigenvalues of $H(\lambda)=-\Delta+\lambda V$ when $\lambda \rightarrow \lambda_{0} . \lambda_{0}$ is the value at which some discrete eigenvalue $e_{i}(\lambda)$ tends to zero. The main method they used in their paper is the Birman-Schwinger technique.

In order to use the Birman-Schwinger technique to $P(\lambda)$, we need to get the asymptotic expansion of $\left(P_{0}-\alpha\right)^{-1}$ for $\alpha$ near zero, $\alpha<0$, which was studied by Wang [1]. In

\section{Springer}

(c) 2013 Jia and Zhao; licensee Springer. This is an Open Access article distributed under the terms of the Creative Commons Attribution License (http://creativecommons.org/licenses/by/2.0), which permits unrestricted use, distribution, and reproduction in any medium, provided the original work is properly cited. 
this paper, we first show that there exists some $\lambda_{0}$ such that when $\lambda>\lambda_{0}, P(\lambda)$ has discrete eigenvalues. Then, we define the Birman-Schwinger kernel $K(\alpha)$ for $P(\lambda)$ and find that there is one-to-one correspondence between the discrete eigenvalues of $K(\alpha)$ and the discrete eigenvalues of $P(\lambda)$. Hence, the asymptotic expansion of the discrete eigenvalue of $P(\lambda)$ can be got through the asymptotic expansion of the discrete eigenvalue of $K(\alpha)$. In our main results, we need to use that $K(\alpha)$ is a bounded operator from $L^{2}$ to $L^{2}$. To get that, we add a strong condition on $V$ (i.e., $\rho_{0}>6$ in (1)). We show that $K(\alpha)$ is a family of compact operators converging to $K(0)$ and obtain the asymptotic expansions of the discrete eigenvalues of $K(\alpha)$ by functional calculus. After that, the convergence rate of the smallest discrete eigenvalue of $P(\lambda)$ is obtained.

Here is the plan of our work. In Section 2, we recall some results of $P_{0}$ and define the Birman-Schwinger kernel $K(\alpha)$ for $P(\lambda)$. The relationship between the eigenvalues of these two kinds of operators is studied. In Section 3, we first study the asymptotic behavior of the discrete eigenvalues of $K(\alpha)$. Then the convergence rate of the smallest discrete eigenvalue of $P(\lambda)$ is obtained. We get the leading term and the estimate of the remainder term of the smallest discrete eigenvalue.

Let us introduce some notations first.

Notation The scalar product on $L^{2}\left(\mathbb{R}^{+} ; r^{d-1} d r\right)$ and $L^{2}\left(\mathbb{R}^{d}\right)$ is denoted by $\langle\cdot, \cdot\rangle$ and that on $L^{2}\left(\mathbb{S}^{d-1}\right)$ by $(\cdot, \cdot) . H^{r, s}\left(\mathbb{R}^{d}\right), r \in \mathbb{Z}, s \in \mathbb{R}$, denotes the weighted Sobolev space of order $r$ with volume element $\langle x\rangle^{2 s} d x$. The duality between $H^{1, s}$ and $H^{-1,-s}$ is identified with the $L^{2}$ product. Denote $H^{0, s}=L^{2, s}$. Notation $\mathcal{L}\left(H^{r, s}, H^{r^{\prime}, s^{\prime}}\right)$ stands for the space of continuous linear operators from $H^{r, s}$ to $H^{r^{\prime}, s^{\prime}}$. The complex plane $\mathbb{C}$ is slit along positive real axis so that $z^{v}=e^{\nu \ln z}$ and $\ln z=\ln |z|+i \arg z$ with $0<\arg z<2 \pi$ are holomorphic there.

\section{Some results for $P_{0}$}

Assume that $(r, \theta)$ are the polar coordinates on $\mathbb{R}^{d}$. Then the condition

$$
-\Delta_{s}+q(\theta)>-\frac{1}{4}(d-2)^{2} \quad \text { on } L^{2}\left(\mathbb{S}^{d-1}\right)
$$

implies

$$
-\Delta+\frac{q(\theta)}{r^{2}} \geq 0
$$

in $L^{2}\left(\mathbb{R}^{d}\right)$ (see [1]).

Now, we recall some results on the resolvent and the Schrödinger group for the unperturbed operator $P_{0}$. Let

$$
\sigma_{\infty}=\left\{v ; v=\sqrt{\lambda+\frac{(d-2)^{2}}{4}}, \lambda \in \sigma\left(-\Delta_{s}+q(\theta)\right)\right\} .
$$

Denote

$$
\sigma_{k}=\sigma_{\infty} \cap[0, k], \quad k \in \mathbb{N} .
$$


For $v \in \sigma_{\infty}$, let $n_{v}$ denote the multiplicity of $\lambda_{v}=v^{2}-\frac{(d-2)^{2}}{4}$ as the eigenvalue of $-\Delta_{s}+q(\theta)$. Let $\varphi_{v}^{(j)}, v \in \sigma_{\infty}, 1 \leq j \leq n_{v}$ denote an orthogonal basis of $L^{2}\left(\mathbb{S}^{d-1}\right)$ consisting of eigenfunctions of $-\Delta_{s}+q(\theta)$ :

$$
\left(-\Delta_{s}+q(\theta)\right) \varphi_{v}^{(j)}=\lambda_{\nu} \varphi_{v}^{(j)}, \quad\left(\varphi_{v}^{(i)}, \varphi_{v}^{(j)}\right)=\delta_{i j}
$$

Let $\pi_{v}$ denote the orthogonal projection in $L^{2}\left(\mathbb{S}^{d-1}\right)$ onto the subspace spanned by the eigenfunctions of $-\Delta_{s}+q(\theta)$ associated with the eigenvalue $\lambda_{v}$, and let $\pi_{v}^{(i)}$ denote the orthogonal projection in $L^{2}\left(\mathbb{S}^{d-1}\right)$ onto the eigenfunction $\varphi_{v}^{(i)}$ :

$$
\begin{aligned}
& \pi_{\nu} f=\sum_{j=1}^{n_{v}}\left(f, \varphi_{\nu}^{(j)}\right) \otimes \varphi_{\nu}^{(j)}, \quad f \in L^{2}\left(\mathbb{S}^{n-1}\right), \\
& \pi_{v}^{(i)} f=\left(f, \varphi_{\nu}^{(i)}\right) \otimes \varphi_{\nu}^{(i)}, \quad f \in L^{2}\left(\mathbb{S}^{d-1}\right), 1 \leq i \leq n_{\nu} .
\end{aligned}
$$

Denote for $v \in \sigma_{\infty}$

$$
z_{v}= \begin{cases}z^{v^{\prime}} & \text { if } v \notin \mathbb{N} \\ z \ln z & \text { if } v \in \mathbb{N}\end{cases}
$$

Here $v^{\prime}=v-[v]$, and $[v]$ is the largest integer which is not larger than $v$. For $v>0$, let $[v]_{-}$ be the largest integer strictly less than $v$. When $v=0$, set $[v]_{-}=0$. Define $\delta_{v}$ by $\delta_{v}=1$, if $v \in \sigma_{\infty} \cap \mathbb{N}, \delta_{v}=0$, otherwise. One has $[v]=[v]_{-}+\delta_{v}$.

The following is the asymptotic expansion for the resolvent $R_{0}(z)=\left(P_{0}-z\right)^{-1}$.

Theorem 2.1 (Theorem 2.2 [1]) The following asymptotic expansion holds for $z$ near zero with $\mathfrak{s} z>0$ :

$$
R_{0}(z)=\delta_{0} \ln z G_{0,0} \pi_{0}+\sum_{j=0}^{N} z^{j} F_{j}+\sum_{\nu \in \sigma_{N}} z_{v} \sum_{j=[v]_{-}}^{N-1} z^{j} G_{v, j+\delta_{v}} \pi_{v}+R_{0}^{(N)}(z)
$$

in $\mathcal{L}(-1, s ; 1,-s), s>2 N+1$. Here

$$
\begin{aligned}
& G_{v, j}(r, \tau)= \begin{cases}b_{v^{\prime}, j}(r \tau)^{j+v^{\prime}} f_{j-[v]}\left(r, \tau ; v^{\prime}\right), & v \notin \mathbb{N}, \\
-\frac{(i r \tau)^{j}}{j !} f_{j-[v]}(r, \tau ; 0), & v \in \mathbb{N},\end{cases} \\
& F_{j} \in \mathcal{L}(-1, s ; 1,-s), \quad s>2 j+1, \\
& R_{0}^{(N)}(z)=O\left(|z|^{N+\epsilon}\right) \in \mathcal{L}(-1, s ; 1,-s), \quad s>2 N+1, \epsilon>0 .
\end{aligned}
$$

Here

$$
b_{v^{\prime}, j}=-\frac{i^{j} e^{-i v^{\prime} \pi / 2} \Gamma\left(1-v^{\prime}\right)}{v^{\prime}\left(v^{\prime}+1\right) \cdots\left(v^{\prime}+j\right)}
$$

for $0 \leq v^{\prime}<1$, and

$$
f_{j}(r, \tau, v)=(r \tau)^{-\frac{1}{2}(n-2)} P_{j, v}(\rho)
$$


with $P_{j, v}(\rho)$ a polynomial in $\rho$ of degree $j$ :

$$
P_{j, v}(\rho)=\frac{i^{j} a_{v}}{j !} \int_{-1}^{1}\left(\rho+\frac{1}{2} \theta\right)^{j}\left(1-\theta^{2}\right)^{\nu-\frac{1}{2}} d \theta, \quad a_{v}=-\frac{e^{-i \pi v / 2}}{2^{2 v+1} \pi^{1 / 2} \Gamma(v+1 / 2)} .
$$

First, we show that $P(\lambda)$ has discrete eigenvalues when $\lambda$ is large enough. In fact, we need only to show that there exists a function $\psi \in L^{2}\left(\mathbb{R}^{d}\right)$ such that $\langle\psi, P(\lambda) \psi\rangle<0$.

From the assumption on $V$, we know that there exists a point $x_{0} \in \mathbb{R}^{d}$ such that $V\left(x_{0}\right)=$ $\inf _{x \in \mathbb{R}^{d}} V(x)$. Choose $\delta>0$ small enough such that for all $x \in B\left(x_{0}, \delta\right), V(x)<\frac{1}{2} V\left(x_{0}\right)$. For $\psi \in C_{0}^{\infty}\left(\mathbb{R}^{d}\right),\|\psi(x)\|=1$, supp $\psi \subset B\left(x_{0}, \delta\right)$, one has

$$
\langle\psi, P(\lambda) \psi\rangle=\left\langle\psi, P_{0} \psi\right\rangle+\lambda\langle\psi, V \psi\rangle\left\langle\left\langle\psi, P_{0} \psi\right\rangle+\frac{\lambda}{2} V\left(x_{0}\right)\right.
$$

when $\lambda$ is large enough, one has $\langle\psi, P(\lambda) \psi\rangle<0$. This means that $P(\lambda)$ has discrete eigenvalues when $\lambda$ is large enough.

$P(\lambda)$ has a continuous spectrum $[0, \infty)$ for $\lambda \geq 0$ because $\lim _{|x| \rightarrow \infty} V(x)$ exists and equals zero (see [3]). We know that $\sigma(P(0))=\sigma\left(P_{0}\right)=[0, \infty)$. Hence, from the continuity of a discrete spectrum of $P(\lambda)$, we know that there exists some $\lambda_{0}$ such that when $\lambda>\lambda_{0}, P(\lambda)$ has eigenvalues less than zero, and when $\lambda \leq \lambda_{0}, \sigma(P(\lambda))=[0, \infty)$. So, $P(\lambda)$ has an eigenvalue $e_{1}(\lambda)<0$ at the bottom of its spectrum for $\lambda>\lambda_{0}$. In Section 3 (Proposition 3.1), we prove that $e_{1}(\lambda)$ is simple and the corresponding eigenfunction can be chosen to be positive everywhere. (There are many results about the simplicity of the smallest eigenvalue of the Schrödinger operator without singularity, but there is no result which can be used directly, because the potential we use in this paper has singularity at zero. Theorem XIII.48 [4] can treat the Schrödinger operator with the potential which has singularity at zero, but the positivity of potential is needed. Hence, we give this result.) From the discussion above and the continuity of a discrete spectrum, one has that $e_{1}(\lambda)$ tends to zero at some $\lambda$. The asymptotic behavior of $e_{1}(\lambda)$ is studied in this paper.

To study the eigenvalues of $P(\lambda)$, we first define a family of Birman-Schwinger kernel operators. Let

$$
K(z)=|V|^{1 / 2}\left(P_{0}-z\right)^{-1}|V|^{1 / 2}
$$

for $z \notin \sigma\left(P_{0}\right)$, and

$$
K(0)=|V|^{1 / 2} F_{0}|V|^{1 / 2}
$$

Then we have the following result.

Proposition 2.2 Let $\alpha<0$. Then

(a) Let

$$
\begin{aligned}
& A=\left\{\psi \in L^{2}\left(\mathbb{R}^{d}\right) ;(P(\lambda)-\alpha) \psi=0\right\}, \\
& B=\left\{\phi \in L^{2}\left(\mathbb{R}^{d}\right) ; K(\alpha) \phi=\lambda^{-1} \phi\right\} .
\end{aligned}
$$

Then $|V|^{1 / 2}$ is injective from $A$ to $B$, and $\left(P_{0}-\alpha\right)^{-1}|V|^{1 / 2}$ is injective from $B$ to $A$. 
(b) The multiplicity of $\alpha$ as the eigenvalue of $P(\lambda)$ is exactly the multiplicity of $\lambda^{-1}$ as the eigenvalue of $K(\alpha)$.

Proof

(a) First, we prove that $|V|^{1 / 2}$ is injective from $A$ to $B$. Note that if $\psi \in A$, then

$$
K(\alpha) \phi=\lambda^{-1} \phi
$$

with $\phi=|V|^{1 / 2} \psi$. And if $\phi=0$, then

$$
\psi=-\lambda\left(P_{0}-\alpha\right)^{-1} V \psi=\lambda\left(P_{0}-\alpha\right)^{-1}|V|^{1 / 2} \phi=0 .
$$

It follows that $|V|^{1 / 2}$ is injective from $A$ to $B$.

Next, we show that $\left(P_{0}-\alpha\right)^{-1}|V|^{1 / 2}$ is injective from $B$ to $A$. If $\phi \in B$, then

$$
(P(\lambda)-\alpha) \psi=0, \quad \text { with } \psi=\left(P_{0}-\alpha\right)^{-1}|V|^{1 / 2} \phi .
$$

And if $\psi=0$, then

$$
0=|V|^{1 / 2} \psi=K(\alpha) \phi=\lambda^{-1} \phi .
$$

It follows that $\left(P_{0}-\alpha\right)^{-1}|V|^{1 / 2}$ is injective from $B$ to $A$.

(b) From (a), one has $\operatorname{dim} A=\operatorname{dim} B$. This means that the multiplicity of $\alpha$ as the eigenvalue of $P(\lambda)$ is exactly the multiplicity of $\lambda^{-1}$ as the eigenvalue of $K(\alpha)$.

From the last proposition, we know that there exists one-to-one correspondence between the discrete eigenvalues of $P(\lambda)$ and the discrete eigenvalues of $K(\alpha)$. Hence, we can study the eigenvalues of $K(\alpha)$ first.

\section{Asymptotic expansion of the eigenvalues}

If $P_{0}$ and $V$ are defined as above, we show that if $P_{0}+V$ has the eigenvalue less than zero, then the smallest eigenvalue of $P_{0}+V$ is simple. We use Theorems XIII.44, XIII.45 [4] to prove it.

Proposition 3.1 Suppose $P_{0}+V$ has an eigenvalue at the bottom of its spectrum. Then this eigenvalue is simple and the corresponding eigenfunction can be chosen to be a positive function.

Proof Let $0 \leq \chi(t) \leq 1$ be a smooth nonincreasing function such that $\chi(t)=1$ if $|t|<1$ and $\chi(t)=0$ if $t>2$. Let $\chi_{n}(t)=\chi(t / n)$. Set $V_{n}=\chi_{n}(r) \frac{q(\theta)}{r^{2}}+V-\langle x\rangle, H_{0}=-\Delta+\langle x\rangle, H_{n}=$ $H_{0}+V_{n}, H=P=P_{0}+V$. From the proof of Theorem XIII.47 [4], we know that $e^{-t H_{0}}$ is positivity preserving and $\left\{e^{-t H_{0}}\right\} \cup L^{\infty}$ acts irreducibly on $L^{2}$. Hence, by Theorem XIII.45 [4], if $H_{n}$ converges to $H$ and $H-V_{n}$ converges to $H_{0}$ in the strong resolvent sense, then $e^{-t H}$ is positivity preserving and $\left\{e^{-t H}\right\} \cup L^{\infty}$ acts irreducibly on $L^{2}$. By Theorems XIII.43 and XIII.44 [4], we can get the result. Since $C_{0}^{\infty}\left(\mathbb{R}^{d}\right)$ is the core for all $P_{n}$ and $P$, and for any $\psi \in C_{0}^{\infty}\left(\mathbb{R}^{d}\right), V_{n} \psi \rightarrow\left(\frac{q(\theta)}{r^{2}}+V\right) \psi$ in $L^{2}$, then we have the necessary strong resolvent convergence by Theorem VIII.25(a) [5]. This ends the proof. 
Proposition 3.2 Assume that $0 \notin \sigma_{\infty} . P_{0} F_{0} u=u$ in $H^{-1, s}$ for any $u \in H^{-1, s}, s>1$.

Proof If $u \in H^{-1, s}$, then $F_{0} u \in H^{1,-s}$. For any test function $\phi \in C_{0}^{\infty}\left(\mathbb{R}^{n}\right)$, we have $\left\langle P_{0} F_{0} u, \phi\right\rangle=$ $\left\langle u, F_{0} P_{0} \phi\right\rangle$. If $0 \notin \sigma_{\infty}$, then we have $\lim _{z \rightarrow 0}\left(P_{0}-z\right)^{-1}=F_{0}$ in $H^{-1, s}$ for $\Im z>0$. It follows $\left\langle u, F_{0} P_{0} \phi\right\rangle=\lim _{z \rightarrow 0}\left\langle u,\left(P_{0}-z\right)^{-1} P_{0} \phi\right\rangle=\lim _{z \rightarrow 0}\left\langle u, \phi-z\left(P_{0}-z\right)^{-1} \phi\right\rangle=\langle u, \phi\rangle$ because $\phi$ and $P_{0} \phi$ belong to $H^{-1, s}$. Hence, $P_{0} F_{0} u=u$ in $H^{-1, s}$.

Proposition 3.3 Assume that $0 \notin \sigma_{\infty} . K(\alpha)$ is a compact operator for $\alpha \leq 0$. And $K(\alpha)$ converges to $K(0)$ in operator norm sense.

Proof For $\alpha<0, K(\alpha)=|V|^{1 / 2}\left(P_{0}-\alpha\right)^{-1}|V|^{1 / 2}$. Since $\left(P_{0}-\alpha\right)^{-1}$ is a bounded operator from $L^{2}\left(\mathbb{R}^{d}\right)$ to $H^{1}\left(\mathbb{R}^{d}\right)$, and $V$ is a compact operator from $H^{1}\left(\mathbb{R}^{d}\right)$ to $L^{2}\left(\mathbb{R}^{d}\right)$, then $V\left(P_{0}-\alpha\right)^{-1}$ is a compact operator on $L^{2}\left(\mathbb{R}^{d}\right)$. Using a similar method to that in Proposition 2.2, we can show that $V\left(P_{0}-\alpha\right)^{-1}$ and $K(\alpha)$ have the same non-zero eigenvalues, and for the same eigenvalue $e(\alpha)$, the multiplicity of $e(\alpha)$ as the eigenvalue of $V\left(P_{0}-\alpha\right)^{-1}$ and the multiplicity of $e(\alpha)$ as the eigenvalue of $K(\alpha)$ are the same. Hence, $K(\alpha)$ is a compact operator. Because

$$
K(\alpha)-K(0)=|V|^{1 / 2}\left[\left(P_{0}-\alpha\right)^{-1}-F_{0}\right]|V|^{1 / 2}=|V|^{1 / 2} R_{0}^{(0)}|V|^{1 / 2}
$$

and if $\rho_{0}>2$, then $|V|^{1 / 2} R_{0}^{(0)}|V|^{1 / 2}=o\left(|\alpha|^{\epsilon}\right)$ in $L^{2}\left(\mathbb{R}^{d}\right)$. Hence, $K(\alpha) \rightarrow K(0)$ in operator norm sense as $\alpha \rightarrow 0$. This means that $K(0)$ is a compact operator.

Lemma 3.4 Suppose $A_{1}, A_{2}$ are two bounded self-adjoint operators on a Hilbert space $H$.

Set

$$
\mu_{n}\left(A_{i}\right)=\inf _{\phi_{1}, \ldots, \phi_{n}} \sup _{\|\psi\|=1, \psi \in\left[\phi_{1}, \ldots, \phi_{n}\right]^{\perp}}\left(\psi, A_{i} \psi\right)
$$

then $\left|\mu_{n}\left(A_{1}\right)-\mu_{n}\left(A_{2}\right)\right| \leq\left\|A_{1}-A_{2}\right\|$.

Proof By the definition of $\mu_{n}\left(A_{i}\right)$, one has

$$
\begin{aligned}
& \left|\mu_{n}\left(A_{1}\right)-\mu_{n}\left(A_{2}\right)\right| \\
& \quad=\left|\inf _{\phi_{1}, \ldots, \phi_{n}} \sup _{\|\psi\|=1, \psi \in\left[\phi_{1}, \ldots, \phi_{n}\right]^{\perp}}\left\langle\psi, A_{1} \psi\right\rangle-\inf _{\phi_{1}, \ldots, \phi_{n}} \sup _{\|\psi\|=1, \psi \in\left[\phi_{1}, \ldots, \phi_{n}\right]^{\perp}}\left\langle\psi, A_{2} \psi\right\rangle\right| \\
& =\left|-\sup _{\phi_{1}, \ldots, \phi_{n}}\left(-\sup _{\|\psi\|=1, \psi \in\left[\phi_{1}, \ldots, \phi_{n}\right]^{\perp}}\left\langle\psi, A_{1} \psi\right\rangle\right)+\sup _{\phi_{1}, \ldots, \phi_{n}}\left(-\sup _{\|\psi\|=1, \psi \in\left[\phi_{1}, \ldots, \phi_{n}\right]^{\perp}}\left\langle\psi, A_{2} \psi\right\rangle\right)\right| \\
& \quad \leq\left|\sup _{\phi_{1}, \ldots, \phi_{n}}\left[-\sup _{\|\psi\|=1, \psi \in\left[\phi_{1}, \ldots, \phi_{n}\right]^{\perp}}\left\langle\psi, A_{1} \psi\right\rangle+\sup _{\|\psi\|=1, \psi \in\left[\phi_{1}, \ldots, \phi_{n}\right]^{\perp}}\left\langle\psi, A_{2} \psi\right\rangle\right]\right| \\
& =\sup _{\phi_{1}, \ldots, \phi_{n}}\left|\sup _{\|\psi\|=1, \psi \in\left[\phi_{1}, \ldots, \phi_{n}\right]^{\perp}}\left\langle\psi, A_{1} \psi\right\rangle-\sup _{\|\psi\|=1, \psi \in\left[\phi_{1}, \ldots, \phi_{n}\right]^{\perp}}\left\langle\psi, A_{2} \psi\right\rangle\right| \\
& \leq \sup _{\phi_{1}, \ldots, \phi_{n}} \sup _{\|\psi\|=1,\left[\phi_{1}, \ldots, \phi_{n}\right]^{\perp}}\left|\left\langle\psi,-A_{1} \psi\right\rangle-\left\langle\psi,-A_{2} \psi\right\rangle\right| \\
& \leq\left\|A_{1}-A_{2}\right\| .
\end{aligned}
$$

This ends the proof. 
Lemma 3.5 Suppose $T(\alpha)$ is a family of compact self-adjoint operators on a separable Hilbert space $H$, and $T(\alpha)=T_{0}+o\left(|\alpha|^{\epsilon}\right)$ for $\alpha$ near zero. Set

$$
\mu_{k}(\alpha)=\inf _{\phi_{1}, \ldots, \phi_{k}} \sup _{\|\psi\|=1, \psi \in\left[\phi_{1}, \ldots, \phi_{k}\right] \perp}\langle\psi, T(\alpha) \psi\rangle .
$$

Then:

(a) $\mu_{k}(\alpha)$ is an eigenvalue of $T(\alpha)$, and $\mu_{k}(\alpha)$ converges when $\alpha \rightarrow 0$. Moreover, if $\mu_{k}(\alpha) \rightarrow$ $\mu_{k}$, then $\mu_{k}$ is an eigenvalue of $T_{0}$.

(b) Suppose that $E_{0} \neq 0$ is an eigenvalue of $T_{0}$ of the multiplicity of $m$. Then there are $m$ eigenvalues (counting multiplicity), $E_{k}(\alpha)(1 \leq k \leq m)$, of $T(\alpha)$ near $E_{0}$. Moreover, we can choose $\left\{\phi_{k}(\alpha) ; 1 \leq k \leq m\right\}$ such that $\left(\phi_{k}(\alpha), \phi_{j}(\alpha)\right)=\delta_{k j}(1 \leq k, j \leq m), \phi_{k}(\alpha)$ is the eigenvector of $T(\alpha)$ corresponding to $E_{k}(\alpha)\left(E_{k}(\alpha) \rightarrow E_{0}\right)$, and $\phi_{k}(\alpha)$ converges as $\alpha \rightarrow 0$. If $\phi_{k}(\alpha)$ converges to $\phi_{k}$, then $\phi_{k}$ is the eigenvector of $T_{0}$ corresponding to $E_{0}$.

Proof

(a) By the min-max principle, we know that $\mu_{k}(\alpha)$ is an eigenvalue of $T(\alpha)$. By Lemma 3.4, one has

$$
\left|\mu_{k}(\alpha)-\mu_{k}(0)\right| \leq\left\|T(\alpha)-T_{0}\right\|=O\left(|\alpha|^{\epsilon}\right) .
$$

It follows that $\mu_{k}(\alpha)$ converges to the eigenvalue of $T_{0}$.

(b) Because $T_{0}$ is a compact operator and $E_{0} \neq 0$ is an eigenvalue of $T_{0}$, then $E_{0}$ is a discrete spectrum of $T_{0}$. Then there exists a constant $\delta>0$ small enough such that $T_{0}$ has only one eigenvalue $E_{0}$ in $B\left(E_{0}, \delta\right)\left(=\left\{z \in \mathbb{C} ;\left|z-E_{0}\right|<\delta\right\}\right)$. For $\alpha$ small enough, $T(\alpha)$ has exactly $m$ eigenvalues (counting multiplicity) in $B\left(E_{0}, \delta\right)$ because the eigenvalues of $T(\alpha)$ converge to the eigenvalues of $T_{0}$ by part (a) of lemma. Suppose the $m$ eigenvalues, near $E_{0}$, of $T(\alpha)$ are $E_{1}(\alpha), E_{2}(\alpha), \ldots, E_{m}(\alpha)$, and the corresponding eigenvectors are $\psi_{1}(\alpha), \psi_{2}(\alpha), \ldots, \psi_{m}(\alpha)$ such that $\left\langle\psi_{k}(\alpha), \psi_{j}(\alpha)\right\rangle=\delta_{k j}$. Let

$$
P_{\alpha}=-\frac{1}{2 \pi i} \oint_{\left|E-E_{0}\right|=\delta}(T(\alpha)-E)^{-1} d E \text {. }
$$

Then $P_{\alpha}=\sum_{k=1}^{m}\left\langle\cdot, \psi_{k}(\alpha)\right\rangle \psi_{k}(\alpha)$. Let $P_{\alpha}^{(k)}=\left\langle\cdot, \psi_{k}(\alpha)\right\rangle \psi_{k}(\alpha)$, then $P_{\alpha}=\sum_{k=1}^{m} P_{\alpha}^{(k)}$. For $\alpha$ near zero, one has

$$
\begin{aligned}
\left\|P_{\alpha}-P_{0}\right\| & =\left\|-\frac{1}{2 \pi i} \oint_{\left|E-E_{0}\right|=\delta}(T(\alpha)-E)^{-1}-\left(T_{0}-E\right)^{-1} d E\right\| \\
& =\left\|-\frac{1}{2 \pi i} \oint_{\left|E-E_{0}\right|=\delta}(T(\alpha)-E)^{-1}\left(T_{0}-T_{\alpha}\right)\left(T_{0}-E\right)^{-1} d E\right\| \\
& =O\left(|\alpha|^{\epsilon}\right) .
\end{aligned}
$$

Let $A=\left\{\phi ;\|\phi\|=1, \phi \in \operatorname{Ran} P_{0}\right\}$. Let $\phi_{k}$ be an element in $A$ such that $\left\|\phi-\psi_{k}(\alpha)\right\|$ acquires the minimum value. Then we have

$$
\begin{aligned}
\left\|P_{\alpha}^{(k)} \phi_{k}-\phi_{k}\right\| & \leq\left\|P_{\alpha}^{(k)} \phi_{k}-\psi_{k}(\alpha)\right\|+\left\|\psi_{k}(\alpha)-\phi_{k}\right\| \\
& =\left\|P_{\alpha}^{(k)} \phi_{k}-P_{\alpha}^{(k)} \psi_{k}(\alpha)\right\|+\left\|\psi_{k}(\alpha)-\phi_{k}\right\| \leq 2\left\|\psi_{k}(\alpha)-\phi_{k}\right\|,
\end{aligned}
$$


and

$$
\begin{aligned}
\left\|\psi_{k}(\alpha)-\phi_{k}\right\| & \leq\left\|\frac{P_{0} \psi_{k}(\alpha)}{\left\|P_{0} \psi_{k}(\alpha)\right\|}-\psi_{k}(\alpha)\right\| \\
& \leq\left\|\frac{P_{0} \psi_{k}(\alpha)}{\left\|P_{0} \psi_{k}(\alpha)\right\|}-P_{0} \psi_{k}(\alpha)\right\|+\left\|P_{0} \psi_{k}(\alpha)-P_{\alpha} \psi_{k}(\alpha)\right\|=O\left(|\alpha|^{\epsilon}\right) .
\end{aligned}
$$

In the last equality, we use the fact

$$
\left\|P_{0} \psi_{k}(\alpha)\right\|=\left\|\left(P_{0} \psi_{k}(\alpha)-\psi_{k}(\alpha)\right)+\psi_{k}(\alpha)\right\|=1+O\left(|\alpha|^{\epsilon}\right)
$$

and

$$
\left\|\frac{P_{0} \psi_{k}(\alpha)}{\left\|P_{0} \psi_{k}(\alpha)\right\|}-P_{0} \psi_{k}(\alpha)\right\|=\left\|P_{0} \psi_{k}(\alpha)\left(\frac{1}{\left\|P_{0} \psi_{k}(\alpha)\right\|}-1\right)\right\|=O\left(|\alpha|^{\epsilon}\right) .
$$

It follows that $\left\|P_{\alpha}^{(k)} \phi_{k}-\phi_{k}\right\|=O\left(|\alpha|^{\epsilon}\right)$. Let $\phi_{k}(\alpha)=\frac{P_{\alpha}^{(k)} \phi_{k}}{\left\|P_{\alpha}^{(k)} \phi_{k}\right\|}$. Then $\left\langle\phi_{k}(\alpha), \phi_{j}(\alpha)\right\rangle=0$ for $k \neq$ $j$ because $P_{\alpha}^{(k)} P_{\alpha}^{(j)}=0$ if $k \neq j$, and $\left\|\phi_{k}(\alpha)-\phi_{k}\right\| \leq\left\|P_{\alpha}^{(k)} \phi_{k}-\phi_{k}\right\|+\left\|\left(1-\frac{1}{\left\|P_{\alpha}^{(k)} \phi_{k}\right\|}\right) P_{\alpha}^{(k)} \phi_{k}\right\|=$ $O\left(|\alpha|^{\epsilon}\right)$. This ends the proof.

Let $0<\alpha_{1}<\alpha_{2}<\cdots<\alpha_{i}<\cdots<\alpha_{m}$ and

$$
T(\beta)=T_{0}+\sum_{i=1}^{m} \beta^{\alpha_{i}}(\ln \beta)^{\delta_{i}} T_{i}+T_{r}(\beta) .
$$

Here, $\delta_{i}=0$ or $1, T_{0} \geq 0, T_{i}(1 \leq i \leq m), T_{r}(\beta)$ are compact operators, and $T_{r}(\beta)=$ $O\left(|\beta|^{\alpha_{m}+\epsilon}\right)$ for $\beta$ near zero. Set

$$
e_{s}=\inf _{\phi_{1}, \ldots, \phi_{s}} \sup _{\|\psi\|=1, \psi \in\left[\phi_{1}, \ldots, \phi_{s}\right]^{\perp}}\left\langle\psi, T_{0} \psi\right\rangle .
$$

Then, by the min-max principle, $e_{s}$ is an eigenvalue of $T_{0}$. Moreover, if $e_{s} \neq 0$, then $e_{s}$ is a discrete eigenvalue of $T_{0}$ because $T_{0}$ is a compact operator. If $e_{s} \neq 0$ is an eigenvalue of $T_{0}$ of multiplicity $m$, without loss, we can suppose that $e_{s}=e_{s+1}=\cdots=e_{s+m-1}$. Then there exist exactly $m$ eigenvalues (counting multiplicity), $e_{s}(\beta), e_{s+1}(\beta), \ldots, e_{s+m-1}(\beta)$, of $T(\beta)$ near $e_{s}$. By Lemma 3.5, we know that there exists a family of normalized eigenvectors $\left\{\phi_{j}(\beta) ; j=\right.$ $s, s+1, \ldots, s+m-1\}$ of $T(\beta)$ such that $T(\beta) \phi_{j}(\beta)=e_{j}(\beta) \phi_{j}(\beta),\left\langle\phi_{j}(\beta), \phi_{k}(\beta)\right\rangle=\delta_{j k}(j, k=$ $s, s+1, \ldots, s+m-1)$, and $\phi_{j}(\beta)(j=s, s+1, \ldots, s+m-1)$ converge as $\beta \rightarrow 0$. Suppose that $\phi_{j}(\beta)$ converge to $\phi_{j}$ for all $j$ such that $e_{j} \neq 0$. Then $\left\langle\phi_{s}, \phi_{j}\right\rangle=\delta_{s j}$. $\left\{\phi_{s}\right\}$ can be extended to a standard orthogonal basis. Set

$$
T_{1}(\beta)=\sum_{s=1}^{m} \beta^{\alpha_{s}}(\ln \beta)^{\delta_{i}} T_{s}+T_{r}(\beta), \quad T_{s j}(\beta)=\left\langle\phi_{s}, T_{1}(\beta) \phi_{j}\right\rangle .
$$

Then we have the following. 
Lemma 3.6 $T(\beta), e_{s}$ are given as before. Then the eigenvalue of $T(\beta), e_{j}(\beta)(j=s, s+1, \ldots, s+$ $m-1)$ has the following form:

$$
e_{j}(\beta)=e_{s}+\frac{\sum_{n=0}^{\infty} a_{n}^{(j)}(\beta)}{\sum_{n=0}^{\infty} b_{n}^{(j)}(\beta)} .
$$

Here

$$
\begin{aligned}
a_{0}^{(j)}(\beta)= & T_{j j}(\beta), \\
a_{1}^{(j)}(\beta)= & -\sum_{\left\{k ; e_{k} \neq e_{s}\right\}}\left(e_{k}-e_{s}\right)^{-1} T_{j k}(\beta) T_{k j}(\beta), \\
a_{2}^{(j)}(\beta)= & \sum_{k \neq j \neq l}\left(e_{k}-e_{s}\right)^{-1}\left(e_{l}-e_{s}\right)^{-1} T_{j k}(\beta) T_{k l}(\beta) T_{l j}(\beta) \\
& -2 \sum_{\left\{k ; e_{k} \neq e_{s}\right\}}\left(e_{k}-e_{s}\right)^{-1} T_{j k}(\beta) T_{k j}(\beta) T_{j j}(\beta), \\
a_{n}^{(j)}(\beta)= & -\frac{(-1)^{n}}{2 \pi i} \oint_{\left|E-e_{s}\right|=\delta}\left(e_{s}-E\right)^{-1} \sum_{i_{1}, i_{2}, \ldots, i_{n}}\left(e_{i_{1}}-E\right)^{-1} \cdots\left(e_{i_{n}}-E\right)^{-1} \\
& \times T_{j i_{1}} T_{i_{1} i_{2}} \cdots T_{i_{n} j} d E \quad \text { for } n>2, \\
b_{0}^{(j)}(\beta)= & 1, \\
b_{1}^{(j)}(\beta)= & 0, \\
b_{2}^{(j)}(\beta)= & \sum_{\left\{k ; e_{k} \neq e_{s}\right\}}\left(e_{s}-e_{k}\right)^{-2} T_{j k}(\beta) T_{k j}(\beta), \\
b_{n}^{(j)}(\beta)= & -\frac{(-1)^{n}}{2 \pi i} \oint_{\left|E-e_{s}\right|=\delta}\left(e_{s}-E\right)^{-2} \sum_{i_{1}, i_{2}, \ldots, i_{n-1}}\left(e_{i_{1}}-E\right)^{-1} \cdots\left(e_{i_{n-1}}-E\right)^{-1} \\
& \times T_{j i_{1}} T_{i_{1} i_{2}} \cdots T_{i_{n-1} j} d E \quad \text { for } n>2 .
\end{aligned}
$$

Proof If $e_{s} \neq 0$, then $e_{s}$ is the discrete eigenvalue of $T_{0}$. Suppose that the multiplicity of $e_{s}$ is $m$, and suppose that $e_{s}=e_{s+1}=\cdots=e_{s+m-1}$ as before. Hence, we can choose $\delta>0$ small enough such that there is only one eigenvalue $e_{s}$ in $B\left(e_{s}, \delta\right)=\left\{z \in \mathbb{C} ;\left|z-e_{s}\right|<\delta\right\}$. We know that $e_{j}(\beta)(j=s, s+1, \ldots, s+m-1)$ converge to $e_{s}$. It follows that if $\delta$ is small enough, there are exactly $m$ eigenvalues (counting multiplicity) of $T(\beta)$ in $B\left(e_{s}, \delta\right)$ for $\beta$ small. Set

$$
P_{\beta} \triangleq-\frac{1}{2 \pi i} \oint_{\left|E-e_{S}\right|=\delta}(T(\beta)-E)^{-1} d E
$$

Then

$$
\begin{aligned}
e_{j}(\beta) & =\frac{\left\langle\phi_{j}, T(\beta) P_{\beta} \phi_{j}\right\rangle}{\left\langle\phi_{j}, P_{\beta} \phi_{j}\right\rangle} \\
& =\frac{\left\langle\phi_{j}, T_{0} P_{\beta} \phi_{j}\right\rangle}{\left\langle\phi_{j}, P_{\beta} \phi_{j}\right\rangle}+\frac{\left\langle\phi_{j}, T_{1}(\beta) P_{\beta} \phi_{j}\right\rangle}{\left\langle\phi_{j}, P_{\beta} \phi_{j}\right\rangle} \\
& =e_{s}+\frac{\left\langle\phi_{j}, T_{1}(\beta) P_{\beta} \phi_{j}\right\rangle}{\left\langle\phi_{j}, P_{\beta} \phi_{j}\right\rangle} .
\end{aligned}
$$


Since

$$
\begin{aligned}
(T(\beta)-E)^{-1} & =\left(T_{0}-E\right)^{-1}\left(I+T_{1}(\beta)\left(T_{0}-E\right)^{-1}\right)^{-1} \\
& =\left(T_{0}-E\right)^{-1} \sum_{n=0}^{\infty}(-1)^{n}\left[T_{1}(\beta)\left(T_{0}-E\right)^{-1}\right]^{n},
\end{aligned}
$$

then

$$
\left\langle\phi_{j}, P_{\beta} \phi_{j}\right\rangle=-\frac{1}{2 \pi i} \oint_{\left|E-e_{s}\right|=\delta}\left\langle\phi_{j},\left(T_{0}-E\right)^{-1} \sum_{n=0}^{\infty}(-1)^{n}\left[\left(T_{1}(\beta)\right)\left(T_{0}-E\right)^{-1}\right]^{n} \phi_{j}\right\rangle d E .
$$

Then

$$
b_{n}^{(j)}(\beta)=-\frac{(-1)^{n}}{2 \pi i} \oint_{\left|E-e_{s}\right|=\delta}\left\langle\phi_{j},\left(T_{0}-E\right)^{-1}\left[\left(T_{1}(\beta)\right)\left(T_{0}-E\right)^{-1}\right]^{n} \phi_{j}\right\rangle d E .
$$

In particular,

$$
\begin{aligned}
b_{0}^{(j)}(\beta)= & -\frac{1}{2 \pi i} \oint_{\left|E-e_{s}\right|=\delta}\left\langle\phi_{j},\left(T_{0}-E\right)^{-1} \phi_{j}\right\rangle d E=1, \\
b_{1}^{(j)}(\beta)= & -\frac{-1}{2 \pi i} \oint_{\left|E-e_{s}\right|=\delta}\left\langle\phi_{j},\left(T_{0}-E\right)^{-1}\left[\left(T_{1}(\beta)\right)\left(T_{0}-E\right)^{-1}\right] \phi_{j}\right\rangle d E \\
= & -\frac{-1}{2 \pi i} \oint_{\left|E-e_{s}\right|=\delta}\left(e_{s}-E\right)^{-2}\left\langle\phi_{j}, T_{1}(\beta) \phi_{j}\right\rangle d E=0, \\
b_{2}^{(j)}(\beta)= & -\frac{(-1)^{2}}{2 \pi i} \oint_{\left|E-e_{s}\right|=\delta}\left\langle\phi_{j},\left(T_{0}-E\right)^{-1}\left[\left(T_{1}(\beta)\right)\left(T_{0}-E\right)^{-1}\right]^{2} \phi_{j}\right\rangle d E \\
= & -\frac{1}{2 \pi i} \oint_{\left|E-e_{s}\right|=\delta}\left(e_{s}-E\right)^{-2}\left\langle\phi_{j},\left[\left(T_{1}(\beta)\right)\left(T_{0}-E\right)^{-1}\left(T_{1}(\beta)\right)\right] \phi_{j}\right\rangle d E \\
= & -\frac{1}{2 \pi i} \oint_{\left|E-e_{s}\right|=\delta}\left(e_{s}-E\right)^{-2} \sum_{k}\left\langle\phi_{j},\left(T_{1}(\beta)\right) \phi_{k}\right\rangle\left(e_{k}-E\right)^{-1}\left\langle\phi_{j},\left(T_{1}(\beta)\right) \phi_{k}\right\rangle d E \\
= & -\frac{1}{2 \pi i} \oint_{\left|E-e_{s}\right|=\delta}\left(e_{s}-E\right)^{-2} \sum_{\left\{k ; e_{k} \neq e_{s}\right\}}\left\langle\phi_{j},\left(T_{1}(\beta)\right) \phi_{k}\right\rangle\left(e_{k}-E\right)^{-1}\left\langle\phi_{j},\left(T_{1}(\beta)\right) \phi_{k}\right\rangle d E \\
& -\frac{1}{2 \pi i} \oint_{\left|E-e_{s}\right|=\delta}\left(e_{s}-E\right)^{-2} \sum_{\left\{k ; e_{k}=e_{s}\right\}}\left\langle\phi_{j},\left(T_{1}(\beta)\right) \phi_{k}\right\rangle\left(e_{k}-E\right)^{-1}\left\langle\phi_{j},\left(T_{1}(\beta)\right) \phi_{k}\right\rangle d E \\
= & \sum_{\left\{k ; e_{k} \neq e_{s}\right\}}\left(e_{s}-e_{k}\right)^{-2} T_{j k}(\beta) T_{k j}(\beta) .
\end{aligned}
$$

In the last step, we use that

$$
\begin{aligned}
\sum_{\left\{k ; e_{k} \neq e_{s}\right\}}\left|\left(e_{k}-e_{j}\right)^{-2} T_{j k}(\beta) T_{k j}(\beta)\right| & \leq C \sum_{\left\{k ; e_{k} \neq e_{s}\right\}}\left(\left|T_{j k}(\beta)\right|^{2}+\left|T_{k j}(\beta)\right|^{2}\right) \\
& \leq C\left(\left\|T_{1}^{*}(\beta) \phi_{j}\right\|^{2}+\left\|T_{1}(\beta) \phi_{j}\right\|^{2}\right) .
\end{aligned}
$$


Similarly, we can get

$$
\begin{aligned}
b_{n}^{(j)}(\beta)= & -\frac{(-1)^{n}}{2 \pi i} \oint_{\left|E-e_{s}\right|=\delta}\left(e_{s}-E\right)^{-2} \sum_{i_{1}, i_{2}, \ldots, i_{n-1}}\left(e_{i_{1}}-E\right)^{-1} \cdots\left(e_{i_{n-1}}-E\right)^{-1} \\
& \times T_{j i_{1}} T_{i_{1} i_{2}} \cdots T_{i_{n-1} j} d E
\end{aligned}
$$

and

$$
\begin{aligned}
a_{0}^{(j)}(\beta)= & T_{j j}(\beta), \\
a_{1}^{(j)}(\beta)= & -\sum_{\left\{k ; e_{k} \neq e_{s}\right\}}\left(e_{k}-e_{s}\right)^{-1} T_{j k}(\beta) T_{k j}(\beta), \\
a_{2}^{(j)}(\beta)= & \sum_{\left\{l, k e_{k} \neq e_{j} \neq e_{l}\right\}}\left(e_{k}-e_{s}\right)^{-1}\left(e_{l}-e_{s}\right)^{-1} T_{j k}(\beta) T_{k l}(\beta) T_{l j}(\beta) \\
& -2 \sum_{\left\{k ; e_{k} \neq e_{s}\right\}}\left(e_{k}-e_{s}\right)^{-1} T_{j k}(\beta) T_{k j}(\beta) T_{j j}(\beta), \\
a_{n}^{(j)}(\beta)= & -\frac{(-1)^{n}}{2 \pi i} \oint_{\left|E-e_{s}\right|=\delta}\left\langle\phi_{j},\left[\left(T_{1}(\beta)\right)\left(T_{0}-E\right)^{-1}\right]^{n+1} \phi_{j}\right\rangle d E \\
= & -\frac{(-1)^{n}}{2 \pi i} \oint_{\left|E-e_{s}\right|=\delta}\left(e_{s}-E\right)^{-1}\left\langle\phi_{j},\left[\left(T_{1}(\beta)\right)\left(T_{0}-E\right)^{-1}\right]^{n} T_{1}(\beta) \phi_{j}\right\rangle d E \\
= & -\frac{(-1)^{n}}{2 \pi i} \oint_{\left|E-e_{s}\right|=\delta}\left(e_{s}-E\right)^{-1} \sum_{i_{1}}\left\langle\phi_{j},\left[\left(T_{1}(\beta)\right)\left(T_{0}-E\right)^{-1}\right]^{n-1}\right. \\
& \left.\times T_{1}(\beta) \phi_{i_{1}}\right\rangle\left(e_{s}-E\right)^{-1} T_{i_{1} j}(\beta) d E \\
= & \cdots \\
= & -\frac{(-1)^{n}}{2 \pi i} \oint_{\left|E-e_{s}\right|=\delta}\left(e_{s}-E\right)^{-1} \sum_{i_{1}, i_{2}, \ldots, i_{n}}\left(e_{i_{1}}-E\right)^{-1} \ldots\left(e_{i_{n}}-E\right)^{-1} \\
& \times T_{j i_{1}} T_{i_{1} i_{2}} \ldots T_{i_{n} j} d E .
\end{aligned}
$$

First, we study the asymptotic expansion of the smallest eigenvalue $e_{1}(\lambda)$ of $P(\lambda)$. By Proposition 3.1, we know that $e_{1}(\lambda)$ is a simple eigenvalue of $P(\lambda)$, and the corresponding eigenfunction can be chosen to be positive. We suppose $u(\lambda)$ is a positive eigenfunction corresponding to $e_{1}(\lambda)$. Then $\tilde{u}(\lambda)=|V|^{1 / 2} u(\lambda) \in L^{2}\left(\mathbb{R}^{d}\right)$. Without loss of generality, we can suppose that $\|\tilde{u}(\lambda)\|_{L^{2}\left(\mathbb{R}^{d}\right)}=1$. Then we can get the following result.

Lemma 3.7 Assume that $0 \notin \sigma_{\infty}$. Set $v_{0}=\min \left\{v ; v \in \sigma_{\infty}\right\}$. $\tilde{u}$ is defined as above. Then $\tilde{u}(\lambda)$ converges in $L^{2}\left(\mathbb{R}^{d}\right)$ when $\lambda \rightarrow \lambda_{0}$. If $v_{0}<1$ and $\tilde{u}(\lambda)$ converges to $\phi$, then $\phi$ is the eigenfunction of $K(0)$, and $\left\langle\phi,|V|^{1 / 2} G_{\nu_{0}, 0} \pi_{\nu_{0}}|V|^{1 / 2} \phi\right\rangle \neq 0$.

Proof By the assumption of $\tilde{u}(\lambda)$, one has $K\left(e_{1}(\lambda)\right) \tilde{u}(\lambda)=\lambda^{-1} \tilde{u}(\lambda)$. One can check that $\tilde{u}(\lambda)$ converges in $L^{2}\left(\mathbb{R}^{d}\right)$ as $\lambda \rightarrow \lambda_{0}$ by Lemma 3.5. And also, by Lemma 3.5, we know that $\phi$ is the normalized eigenfunction of $K(0)$ corresponding to $E_{0} . \phi$ is a positive function since $\tilde{u}(\lambda)$ is a positive function. Let $u=F_{0}|V|^{1 / 2} \phi$, then $P\left(\lambda_{0}\right) u=0$ and $u$ is a positive function 
because $|V|^{1 / 2} u=|V|^{1 / 2} F_{0}|V|^{1 / 2} \phi=K(0) \phi=\lambda_{0}^{-1} \phi$. Then

$$
\begin{aligned}
\langle\phi & \left.|V|^{1 / 2} G_{v_{0}, 0} \pi_{v_{0}}|V|^{1 / 2} \phi\right\rangle \\
& =\lambda_{0}^{2}\left\langle|V|^{1 / 2} u,|V|^{1 / 2} G_{v_{0}, 0} \pi_{v_{0}}|V|^{1 / 2}|V|^{1 / 2} u\right\rangle \\
& =\lambda_{0}^{2}\left\langle V u, G_{v_{0}, 0} \pi_{v_{0}} V u\right\rangle \\
& =\lambda_{0}^{2} C_{v_{0}}\left|\left\langle V u,|y|^{-\frac{n-2}{2}+v_{0}} \varphi_{\nu_{0}}\right\rangle\right|^{2} \\
& \neq 0 .
\end{aligned}
$$

In the last equality, we use the fact that

$$
G_{v_{0}, 0}=(r \tau)^{v_{0}} b_{v_{0}, 0} f_{0}=d_{v_{0}} b_{v_{0}, 0}(r \tau)^{-\frac{d-2}{2}+v_{0}}=C_{v_{0}}(r \tau)^{-\frac{d-2}{2}+v_{0}}
$$

with $d_{\nu_{0}}=-\frac{e^{-\frac{1}{2} i \pi v_{0}}}{2^{2 v_{0}+1} \Gamma\left(v_{0}+1\right)}$, and $C_{\nu_{0}}=d_{\nu_{0}} b_{\nu_{0}, 0}$. This ends the proof.

Theorem 3.8 Assume that $0 \notin \sigma_{\infty}$. $\phi$ is defined in Lemma 3.7. If $\rho_{0}>6$, one of three exclusive situations holds:

(a) If $\sigma_{1}=\emptyset$, then

$$
e_{1}(\lambda)=-c\left(\lambda-\lambda_{0}\right)+o\left(\lambda-\lambda_{0}\right)
$$

with $c=\left(\lambda_{0}\left\|F_{0}|V|^{\frac{1}{2}} \phi\right\|\right)^{-2} \neq 0$.

(b) If $v_{0}=1$, then

$$
e_{1}(\lambda)=-c \frac{\lambda-\lambda_{0}}{\ln \left(\lambda-\lambda_{0}\right)}+o\left(\frac{\lambda-\lambda_{0}}{\ln \left(\lambda-\lambda_{0}\right)}\right)
$$

with $c=\lambda_{0}^{-2}\left\langle\phi,|V|^{1 / 2} G_{1,0} \pi_{1}|V|^{1 / 2} \phi\right\rangle^{-1} \neq 0$.

(c) If $v_{0}<1$, then

$$
e_{1}(\lambda)=c\left(\left(\lambda-\lambda_{0}\right)^{\frac{1}{\nu_{0}}}\right)+o\left(\left(\lambda-\lambda_{0}\right)^{\frac{1}{\nu_{0}}}\right)
$$

with $c=\lambda_{0}^{-2}\left\langle\phi,|V|^{1 / 2} G_{\nu_{0}, 0} \pi_{\nu_{0}}|V|^{1 / 2} \phi\right\rangle^{-1} \neq 0$.

Proof

(a) By Theorem 2.1, one has

$$
R_{0}(\alpha)=F_{0}+\alpha F_{1}+R_{0}^{(1)}(\alpha), \quad \text { in } \mathcal{L}(-1, s ; 1,-s), s>3
$$

Then if $\rho_{0}>6$, we can get $K(\alpha)=K(0)+|V|^{1 / 2}\left(\alpha F_{1}+R_{0}^{(1)}(\alpha)\right)|V|^{1 / 2}$ in $\mathcal{L}(0,0 ; 0,0)$. Because $e_{1}(\lambda)$ is the simple eigenvalue of $P(\lambda)$, then $\lambda^{-1}$ is the simple eigenvalue of $K\left(e_{1}(\lambda)\right)$. Since $V \leq 0$, one has that $P(\lambda)$ is monotonous with respect to $\lambda$ and so is the $e_{1}(\lambda)$. Hence, $K\left(e_{1}(\lambda)\right)$ and the eigenvalues of $K\left(e_{1}(\lambda)\right)$ are monotonous with respect to $\lambda$. Therefore, we have that $\lambda^{-1}$ is the biggest eigenvalue of $K\left(e_{1}(\lambda)\right)$. If not, suppose that $a>\lambda^{-1}$ is an eigenvalue of $K\left(e_{1}(\lambda)\right)$, then by the continuity and monotony of the eigenvalue of $K\left(e_{1}(\lambda)\right)$ with respect to $\lambda$, we know that there exists a constant $\lambda^{\prime}<\lambda$ such that $\lambda \in \sigma\left(K\left(e_{1}\left(\lambda^{\prime}\right)\right)\right)$. 
It follows that $e_{1}\left(\lambda^{\prime}\right)<e_{1}(\lambda)$ is an eigenvalue of $P_{0}+\lambda V$. This is contradictory to that $e_{1}(\lambda)$ is the smallest eigenvalue. By Lemma 3.7, we know the normalized eigenfunction $\tilde{u}(\lambda)$ of $K\left(e_{1}(\lambda)\right)$ converges to $\phi$. It follows $\tilde{u}(\lambda)=\frac{P_{\lambda} \phi}{\left\|P_{\lambda} \phi\right\|}$ with $P_{\lambda}=-\frac{1}{2 \pi i} \oint_{\left|E-E_{0}\right|=\delta}\left(K\left(e_{1}(\lambda)\right)-E\right)^{-1} d E$. Then

$$
\mu\left(e_{1}(\lambda)\right)=\left\langle\tilde{u}(\lambda), K\left(e_{1}(\lambda)\right) \tilde{u}(\lambda)\right\rangle=\frac{\left\langle\phi, K\left(e_{1}(\lambda)\right) P_{\lambda} \phi\right\rangle}{\left\langle\phi, P_{\lambda} \phi\right\rangle} .
$$

Here $\mu\left(e_{1}(\lambda)\right)$ is the eigenvalue of $K\left(e_{1}(\lambda)\right)$ corresponding to the eigenfunction $\tilde{u}(\lambda)$. By Lemma 3.6, we should compute $\left\langle\phi,|V|^{1 / 2} F_{1}|V|^{1 / 2} \phi\right\rangle$. Let $\psi=F_{0}|V|^{1 / 2} \phi$. From the definition of $\phi$, one has $K(0) \phi=\lambda_{0}^{-1} \phi$. Hence,

$$
\left(P_{0}+\lambda_{0} V\right) \psi=P_{0} F_{0}|V|^{1 / 2} \phi+\lambda_{0} V F_{0}|V|^{1 / 2} \phi=0
$$

In the last equality, we use the fact $P_{0} F_{0}|V|^{1 / 2} \phi=|V|^{1 / 2} \phi$, which can be obtained by Proposition 3.2. Since $v_{0}>1$, we have $\psi \in L^{2}\left(\mathbb{R}^{d}\right)$ by Theorem 3.1 [1]. So, $\psi$ is the ground state of $P\left(\lambda_{0}\right)$. We also have

$$
\begin{aligned}
& |V|^{1 / 2} \psi=K(0) \phi=\lambda_{0}^{-1} \phi, \\
& \left(P_{0}-\alpha\right)^{-1}|V| \psi=\lambda_{0}^{-1}\left(\psi+\alpha R_{0}(\alpha) \psi\right) .
\end{aligned}
$$

Hence,

$$
\begin{aligned}
|V|^{1 / 2} F_{1}|V|^{1 / 2} \phi & =\lambda_{0}|V|^{1 / 2} F_{1}|V|^{1 / 2}|V|^{1 / 2} \psi \\
& =\lambda_{0} \alpha^{-1}|V|^{1 / 2}\left(R_{0}(\alpha)-F_{0}\right)|V| \psi+O\left(|\alpha|^{\epsilon}\right) \\
& =\lambda_{0} \alpha^{-1}|V|^{1 / 2} \lambda_{0}^{-1}\left(\psi+\alpha R_{0}(\alpha) \psi-\psi\right)+O\left(|\alpha|^{\epsilon}\right) \\
& =|V|^{1 / 2} R_{0}(\alpha) \psi+O\left(|\alpha|^{\epsilon}\right) .
\end{aligned}
$$

It follows

$$
\begin{aligned}
\left\langle\phi,|V|^{1 / 2} F_{1}|V|^{1 / 2} \phi\right\rangle & =\lim _{\alpha \rightarrow 0}\left\langle|V|^{1 / 2} \phi, R_{0}(\alpha) \psi\right\rangle \\
& =\left\langle F_{0}|V|^{1 / 2} \phi, \psi\right\rangle \\
& =\|\psi\|^{2} \neq 0 .
\end{aligned}
$$

So, $\mu(\alpha)=\lambda_{0}^{-1}+c_{1} \alpha+o\left(|\alpha|^{1+\epsilon}\right)$ with $c_{1}=\left\|F_{0}|V|^{\frac{1}{2}} \phi\right\|^{2}$. By the Proposition 2.2, one has $\mu\left(e_{1}(\lambda)\right)=\lambda^{-1}$. It follows

$$
\lambda^{-1}=\lambda_{0}^{-1}+c_{1} e_{1}(\lambda)+O\left(\left|e_{1}(\lambda)\right|^{1+\epsilon}\right) .
$$

Since $\lambda^{-1}=\lambda_{0}^{-1}-\lambda_{0}^{-2}\left(\lambda-\lambda_{0}\right)+O\left(\left|\lambda-\lambda_{0}\right|^{2}\right)$, we can get the leading term of $e_{1}(\lambda)$ is $-c\left(\lambda-\lambda_{0}\right)$ with $c=\left(\lambda_{0}\left\|F_{0}|V|^{\frac{1}{2}} \phi\right\|\right)^{-2}$.

(b) If $v_{0}=1$, then

$$
K(\alpha)=K(0)+\alpha \ln \alpha|V|^{1 / 2} G_{1,0} \pi_{1}|V|^{1 / 2}+O(\alpha) .
$$


By Lemma 3.7, one has

$$
\left\langle\phi,|V|^{1 / 2} G_{1,0} \pi_{1}|V|^{1 / 2} \phi\right\rangle \neq 0 .
$$

Then we have

$$
\mu(\alpha)=\lambda_{0}^{-1}+c_{1} \alpha \ln \alpha+o(\alpha)
$$

with $c_{1}=\left\langle\phi,|V|^{1 / 2} G_{1,0} \pi_{1}|V|^{1 / 2} \phi\right\rangle$. As in (a), using $\mu\left(e_{1}(\lambda)\right)=\lambda^{-1}$ and

$$
\lambda^{-1}=\lambda_{0}^{-1}-\lambda_{0}^{-2}\left(\lambda-\lambda_{0}\right)+O\left(\left|\lambda-\lambda_{0}\right|^{2}\right),
$$

one has $-\lambda^{-2}\left(\lambda-\lambda_{0}\right)+O\left(\left|\lambda-\lambda_{0}\right|^{2}\right)=c e_{1}(\lambda) \ln e_{1}(\lambda)+o\left(e_{1}(\lambda)\right)$. To get the leading term of $e_{1}(\lambda)$, we can suppose that $e_{1}(\lambda)=\left(\lambda-\lambda_{0}\right) f(\lambda)$. Then, by comparing the leading term, we can get $f(\lambda)=1 / \ln \left(\lambda-\lambda_{0}\right)$. It follows

$$
e_{1}(\lambda)=-c \frac{\lambda-\lambda_{0}}{\ln \left(\lambda-\lambda_{0}\right)}+o\left(\frac{\lambda-\lambda_{0}}{\ln \left(\lambda-\lambda_{0}\right)}\right)
$$

with $c=\lambda_{0}^{-2}\left\langle\phi,|V|^{1 / 2} G_{1,0} \pi_{1}|V|^{1 / 2} \phi\right\rangle^{-1}$.

(c) If $v_{0}<1$, one has

$$
K(\alpha)=K(0)+\sum_{0<\nu \leq 1} \alpha^{\nu}|V|^{1 / 2} G_{\nu, 0} \pi_{\nu}|V|^{1 / 2}+O(|\alpha|) .
$$

By Lemma 3.7, we know that $\left\langle\phi,|V|^{1 / 2} G_{\nu_{0}, 0} \pi_{\nu_{0}}|V|^{1 / 2} \phi\right\rangle^{-1} \neq 0$. Using the same argument as before, we can conclude

$$
\mu(\alpha)=\lambda_{0}^{-1}+c_{1} \alpha^{\nu_{0}}+o\left(|\alpha|^{\nu_{0}}\right)
$$

with $c_{1}=\left\langle\phi,|V|^{1 / 2} G_{v_{0}, 0} \pi_{v_{0}}|V|^{1 / 2} \phi\right\rangle$. As above, we can get that

$$
e_{1}(\lambda)=c\left(\lambda-\lambda_{0}\right)^{\frac{1}{\nu_{0}}}+o\left(\left(\lambda-\lambda_{0}\right)^{\frac{1}{\nu_{0}}}\right)
$$

with $c=\lambda_{0}^{-2}\left\langle\phi,|V|^{1 / 2} G_{\nu_{0}, 0} \pi_{\nu_{0}}|V|^{1 / 2} \phi\right\rangle^{-1}$.

Competing interests

The authors declare that they have no competing interests.

Authors' contributions

Both authors contributed equally to the manuscript and read and approved the final manuscript.

\section{Author details}

${ }^{1}$ Mathematics and Statistics School, Henan University of Science and Technology, No. 263, Luo-Long District, Kai-Yuan Road, Luoyang City, Henan Province 471023, China. ${ }^{2}$ College of Civil Engineering and Architecture, Zhejiang University, B505 Anzhong Building, 866 Yuhangtang Road, Hangzhou, Zhejiang Province 310058, China.

\section{Acknowledgements}

This research is supported by the Natural Science Foundation of China $(11101127,11271110)$ and the Natural Science Foundation of Educational Department of Henan Province (2011B110014). 
References

1. Wang, XP: Asymptotic expansion in time of the Schrödinger group on conical manifolds. Ann. Inst. Fourier 56 1903-1945 (2006)

2. Klaus, M, Simon, B: Coupling constant thresholds in non-relativistic quantum mechanics, I. Short range two-body systems. Ann. Phys. 130, 251-281 (1980)

3. Berezin, FA, Shubin, MA: The Schrödinger Equation. Kluwer, Dordrecht (1991)

4. Reed, M, Simon, B: Methods of Modern Mathematical Physics, IV. Analysis of Operators. Academic Press, New York (1978)

5. Reed, M, Simon, B: Methods of Modern Mathematical Physics, I. Functional Analysis. Academic Press, New York (1979)

doi:10.1186/1687-2770-2013-62

Cite this article as: Jia and Zhao: Coupling constant limits of Schrödinger operators with critical potentials. Boundary Value Problems 2013 2013:62.

Submit your manuscript to a SpringerOpen ${ }^{\circ}$ journal and benefit from:

- Convenient online submission

Rigorous peer review

- Immediate publication on acceptance

- Open access: articles freely available online

- High visibility within the field

- Retaining the copyright to your article

Submit your next manuscript at $>$ springeropen.com 